\title{
Novel high intensity gamma-source at CERN: the Gamma Factory Initiative
}

\author{
C. Curatolo*1, W. Placzek ${ }^{2}$, M. W. Krasny ${ }^{3}$, L. Serafini ${ }^{4}$, M. Zanetti ${ }^{1,5}$ \\ ${ }^{1}$ INFN - Padova, Padova, Italy \\ ${ }^{2}$ Marian Smoluchowski Institute of Physics Jagiellonian University, Krakow, Poland \\ ${ }^{3}$ LPNHE, Paris, France \\ ${ }^{4}$ INFN - Milano, Milano, Italy \\ ${ }^{5}$ Universitá di Padova, Padova, Italy \\ E-mail: camilla.curatolo@pd.infn.it
}

\begin{abstract}
Collisions of Partially Stripped Ions (PSI) with laser light to produce high intensity gamma-ray beams are the backbone of the Gamma Factory (GF) initiative. The source, if realised at LHC, could significantly push up the intensity limits of the presently operating ones, reaching the flux of the order of $10^{17}$ photons/s, in the particularly interesting gamma-ray energy domain of 1 to $400 \mathrm{MeV}$. The unprecedented-intensity, energy-tuned gamma beams, together with the gammabeams-driven secondary beams of polarized positrons, polarized muons, neutrinos, neutrons and radioactive ions would constitute the basic research tools of the proposed Gamma Factory. We discuss the GF concept and the preliminary estimates of the emitted gamma beams phase spaces given by two newly developed Monte Carlo codes which simulate the PSI-laser interactions.
\end{abstract}

Sixth Annual Conference on Large Hadron Collider Physics (LHCP2018)

4-9 June 2018

Bologna, Italy

${ }^{*}$ Speaker. 


\section{The Gamma Factory proposal}

The Gamma Factory proposal $[1,2]$ is based on the idea to use Partially Stripped Ion (PSI) beams as the drivers of a high intensity gamma-ray source [3]. The PSI beam (beam of ions carrying one or more electrons which have not been stripped along the way from the ion source to the final PSI beam storage ring) collides head-on with a laser. The laser energy is tuned to enable the resonant absorption of the laser photons by the PSI beam which is followed by spontaneous atomic-transition emissions of secondary photons. The initial laser-photon frequency is boosted by a factor of up to $4 \times \gamma_{i}^{2}$, where $\gamma_{i}$ is the Lorenz factor of the partially stripped ion beam. From the kinematic point of view the process is similar to inverse Compton scattering (light sources in the $\mathrm{MeV}$ energy range operating via ICS have already been constructed and are operating in several countries: HI $\gamma$ S-USA, LEPS-Japan, LADON-Italy, ROKK-1-Russia, GRAAL-France and LEGSUSA), moreover the PSI-beam-driven light source intensity could be much higher thanks to the giga-barn resonant absorption cross section (ICS cross section $\sim$ barn).

\section{PSI-laser collision simulations}

A preliminary attempt to simulate the PSI-laser collisions has been performed by modifying the existing Monte Carlo codes Cain and CMCC [4]. Cain, written by K. Yokoya et al. [5], is a stand-alone Monte Carlo program for simulations of beam-beam interactions involving highenergy electrons, positrons and photons. CMCC [6] is a Monte Carlo event generator useful to simulate asymmetric electron-photon or proton-photon collisions [7, 8]. These two codes have been adapted to the new interaction scheme and they have been named respectively GF-CAIN and GF-CMCC. At the moment we have assumed a very short lifetime of the PSI in the excited state, a negligible probability of double photon absorption, a flat differential cross section for the spontaneous emission, a monochromatic laser colliding head-on with the PSI beam. By means of the newly developed GF-CAIN and GF-CMCC, we have simulated the interaction between $X e^{39+}, \mathrm{Pb}^{81+}$ and laser light. The parameters of the two specific examples we have considered are reported in Table 1. For the Xenon partially stripped ions, SPS-like parameters have been adopted, while for the Lead PSI typical LHC parameters have been used. In the first case the collision is performed with a green laser, in the latter case with a free electron laser. All the parameters are purely indicative and we adopted in the $\mathrm{Pb}^{81+}$ example an arbitrarily increased value for the beam emittance ( $9 \mathrm{~mm}$ mad instead of the expected value, to be confirmed in the $2018 \mathrm{~Pb}$ runs, of about $1.3 \mathrm{~mm} \mathrm{mrad}$ ) in order to better illustrate its effect on the energy-angle correlation and on the efficiency of angular beam collimation. The emitted photon beam features simulated by the two codes are presented in Figs. 1 (only GF-CMCC), 2 (GF-CAIN and GF-CMCC). The first column of Fig. 1 shows the angular distribution of the full photon beam: in both cases half of the photons are emitted within a cone of $1 / \gamma_{i}$ aperture around the incoming PSI direction. The lower emittance of the Xenon beam with respect to the Lead one is mapped onto the photons as we can see in the second and third columns of Fig. 1: the energy-angle correlation is shown for different collimation angles $\theta_{\gamma}=15,35,75 \mathrm{mrad}$ in the Xenon case and $\theta_{\gamma}=0.25,0.5,1 \mathrm{mrad}$ for the Lead. The data reported in Fig. 2 have been simulated by with the two independent codes and the comparison of the results is shown for the $P b^{81+}$-FEL collision case. 


\begin{tabular}{ccc}
\hline PSI Beam & $X e^{39+}$ & $P b^{81+}$ \\
\hline$M_{i}$ ion mass & $120 \mathrm{GeV} / c^{2}$ & $193 \mathrm{GeV} / c^{2}$ \\
$E_{i}$ ion energy & $4.19 \mathrm{TeV}$ & $579 \mathrm{TeV}$ \\
$\gamma_{i}=E_{i} / M_{i}$ & 34.66 & 3000 \\
$N_{i}$ ions per bunch & $2 \cdot 10^{9}$ & $9.4 \cdot 10^{7}$ \\
$\Delta \gamma_{i} / \gamma_{i}$ rel. en. spread & $3 \cdot 10^{-4}$ & 0 \\
$\varepsilon^{n}$ norm. trans. emitt. & $2 \mathrm{~mm} \mathrm{mrad}$ & $9 \mathrm{~mm} \mathrm{mrad}$ \\
$\beta_{x}=\beta_{y}$ beta function & $50 \mathrm{~m}$ & $0.5 \mathrm{~m}$ \\
$\sigma_{x}$ rms trans. size & $1.7 \mathrm{~mm}$ & $38.7 \mu \mathrm{m}$ \\
$\sigma_{z}$ rms bunch length & $12 \mathrm{~cm}$ & $15 \mathrm{~cm}$ \\
\hline Laser & $\mathrm{Green}$ & $\mathrm{FEL}$ \\
\hline$\lambda_{L}$ wavelength $\left(E_{L}\right.$ photon energy) & $532 \mathrm{~nm}(2.33 \mathrm{eV})$ & $108.28 \mathrm{~nm}(11.45 \mathrm{eV})$ \\
$N_{L}$ photons per pulse & $8.73 \cdot 10^{14}$ & $3 \cdot 10^{13}$ \\
$U_{L}$ pulse energy & $0.33 \mathrm{~mJ}$ & $56 \mu \mathrm{J}$ \\
$P_{L}$ mean power (rep. rate $\left.40 \mathrm{MHz}\right)$ & $13.2 \mathrm{~kW}$ & $2.24 \mathrm{~kW}$ \\
$w_{0}$ waist at IP $\left(2 \sigma_{L}\right)$ & $3.4 \mathrm{~mm}$ & $50.84 \mu \mathrm{m}$ \\
$R_{L}$ Rayleigh length & $68.23 \mathrm{~m}$ & $7.5 \mathrm{~cm}$ \\
$\sigma_{t}$ rms pulse length & $1 \mathrm{~m}$ & $15 \mathrm{~cm}$ \\
\hline$\gamma$ photons & $161.5 \mathrm{eV}$ & $68.7 \mathrm{keV}$ \\
\hline$E_{\text {res }}=E_{L}^{\prime}$ resonance energy & $11.2 \mathrm{keV}$ & $412 \mathrm{MeV}$ \\
\hline$E_{\gamma}^{\text {max }}$ maximum photon energy &
\end{tabular}

Table 1: Simulation parameters for $X e^{39+}$ and $P b^{81+}$-laser collisions.
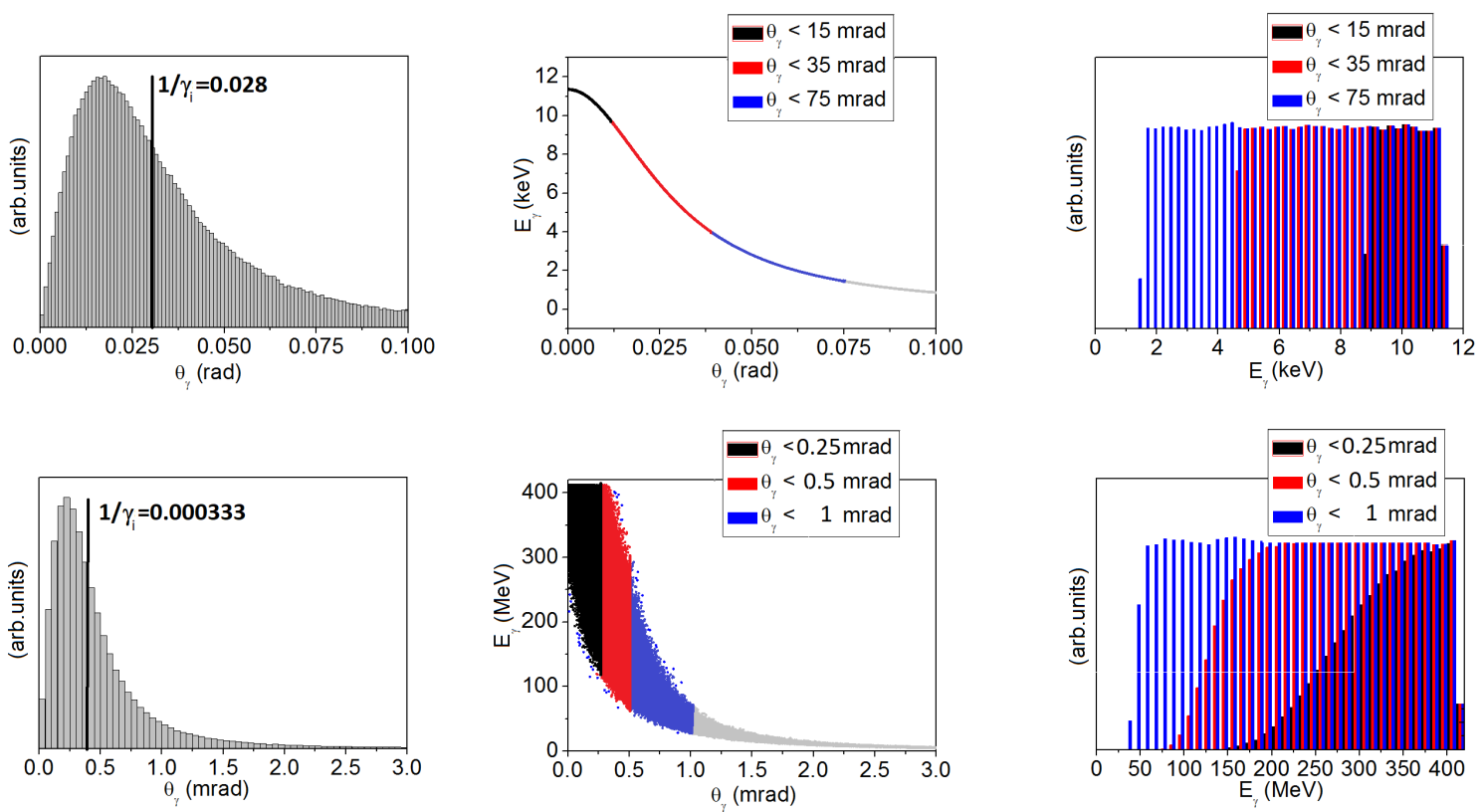

Figure 1: Features of secondary photons emitted by $X e^{39+}$-laser collision first row and $P b^{81+}$-FEL second row, simulation with GF-CMCC. First column: angular distribution of the full emitted photon beam and $1 / \gamma_{i}$ value reported on the graph. Second column: energy as a function of the emission angle, colours represent different collimation angles. Third column: energy distribution for three possible collimated beams. 

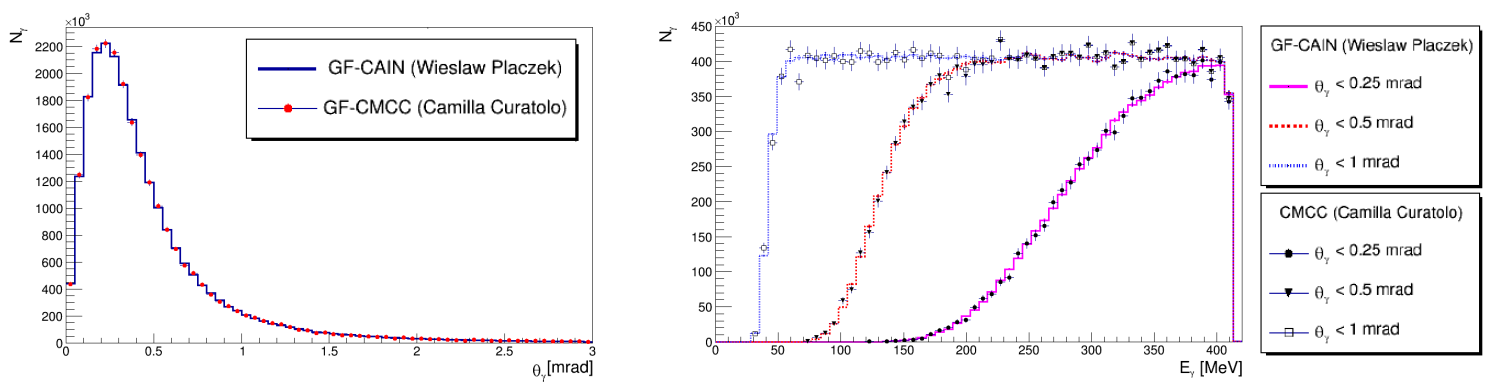

Figure 2: GF-CAIN and GF-CMCC results comparison in case $P b^{81+}$-FEL collision. Left: angular distribution of the full emitted photon beam. Right: spectrum of the emitted photon beam collimated at $\theta_{\gamma}=0.25,0.5,1 \mathrm{mrad}$.

\section{PSI beams at CERN}

The first important step towards the GF is to analyze the storage stability of the PSI beams. It remains to be stressed that a large fraction of the beam cooling and beam manipulation techniques exploiting the internal degrees of freedom of the beam particles, which have been mastered over three decades by the atomic physics community, could be directly applied to the high energy PSI beams. After a first attempt carried out at SPS with $\mathrm{Xe}^{39+}$, on the 25 th of July this year $\mathrm{Pb}^{81+}$ beam has been injected in the LHC. The $\mathrm{Pb}^{81+}$ beam lifetime was measured to be $\sim 38$ hours, a very impressive result (see Fig. 3).

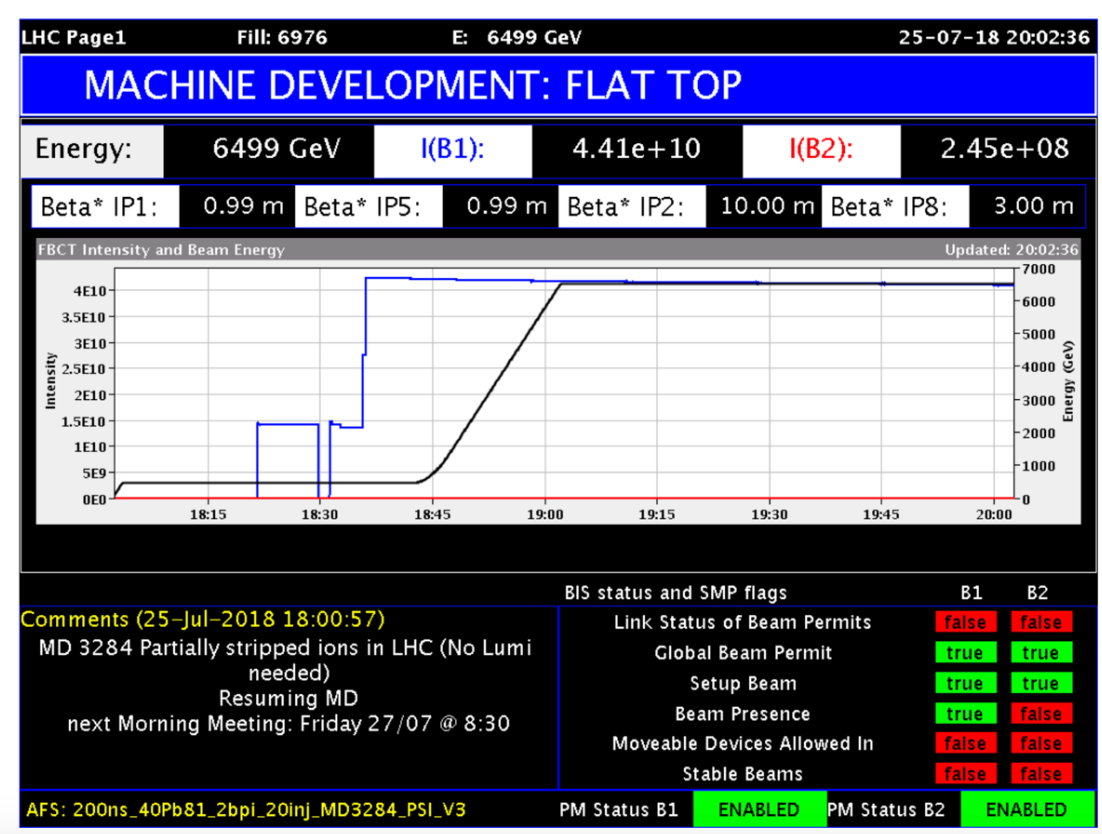

Figure 3: Screenshot of the LHC monitor: $\mathrm{Pb}^{81+}$ bunch trains $\left(4.4 \times 10^{10}\right.$ total charges $)$ ramped to the maximal LHC energy of $6.5 \mathrm{TeV}$ proton equivalent observed for about two hours. 


\section{Future plans}

The unprecedented-intensity, energy-tuned, gamma beams, together with the gamma-beamsdriven secondary beams of polarized positrons, polarized muons, neutrinos, neutrons and radioactive ions would constitute the basic research tools of the proposed Gamma Factory. A broad spectrum of new opportunities, in a vast domain of uncharted fundamental and applied physics territories, could be opened by the Gamma Factory research programme.

We have discussed here our first attempt to simulate PSI-laser collisions. The simulations for $X e^{39+}, P b^{81+}$ and laser light have been performed with the two independent codes GF-CAIN and GF-CMCC and the results are in very good agreement. Nevertheless, these results are still very preliminary since many important approximations have been done: the details of the interaction have to be considered more carefully, we have to insert the correct density, spectrum and temporal shape of the incoming photon beam in order to have a reliable estimation of the total number of emitted photons. Moreover, the incoming beams parameters have to be optimized and the interaction region geometry remains to be designed.

Tests to understand the storage stability of the PSI beams have been carried out at the LHC showing a very long lifetime. To complete the feasibility proof, a "proof-of-principle" SPS experiment should be performed.

The presented above research option for CERN may turn out not only to be scientifically attractive but also cost-effective because it proposes to re-use, in a novel manner, the existing CERN accelerator infrastructure. It may be considered as complementary to the present hadron-collision programme and could be performed at any stage of the LHC lifetime. It is necessary to perform a detailed validation of the achievable performances of the Gamma Factory initiative for each branch of its application domains, to build up the physics case for its research programme and, most importantly, to attract a wide community to this initiative.

\section{References}

[1] M. W. Krasny, The Gamma Factory proposal for CERN, arxiv:1511.07794 (2015)

[2] M. W. Krasny et al., The CERN Gamma Factory initiative: an ultra-high intensity gamma source in Proc. 9th Int. Particle Accelerator Conf. (IPAC'18), Vancouver, BC, Canada, WEYGBD3 (2018)

[3] E. G. Bessonov, Light sources based on relativistic ion beams, Nucl. Instr. Meth. Phys. Res. B 309 (2013) 92-94 and the references quoted therein.

[4] C. Curatolo, W. Placzek, L. Serafini, and M. W. Krasny, New simulation programs for partially stripped ions - laser light collisions in Proc. 9th Int. Particle Accelerator Conf. (IPAC'18), Vancouver, BC, Canada, THPMF076 (2018)

[5] K. Yokoya, User manual of CAIN, version 2.42, https://ilc.kek.jp/ yokoya/CAIN/Cain242/ (2011)

[6] C. Curatolo, PhD Thesis: High brilliance photon pulses interacting with relativistic electron and proton beams, https://air.unimi.it/handle/2434/358227 (2016)

[7] C. Curatolo, F. Broggi and L. Serafini, Phase space analysis of secondary beams generated in hadron-photon collisions, in press on Nucl. Instr. Meth. Phys. Res. A

[8] L. Serafini, F. Broggi and C. Curatolo, Production of TeV-class photons via Compton back-scattering on proton beams of a keV high brilliance FEL, Nucl. Instr. Meth. Phys. Res., Sect. B 402, 343 (2017) 